\title{
A score including ADAM17 substrates correlates to recurring cardiovascular event in subjects with atherosclerosis
}

\author{
Rizza Stefano ${ }^{\mathrm{a}, \mathrm{b}}$, Copetti Massimiliano ${ }^{\mathrm{c}}$, Cardellini Marina ${ }^{\mathrm{a}, \mathrm{b}}$, Menghini Rossella ${ }^{\mathrm{a}}$, \\ Pecchioli Chiara $^{\mathrm{a}, \mathrm{b}}$, Luzi Alessio ${ }^{\mathrm{a}, \mathrm{b}}$, Di Cola Giovanni ${ }^{\mathrm{a}, \mathrm{b}}$, Porzio Ottavia ${ }^{\mathrm{d}}$, \\ Ippoliti Arnaldo e, Romeo Franco a , Pellegrini Fabio ${ }^{c}$, Federici Massimo a,b,* \\ a Department of Systems Medicine, University of Rome Tor Vergata, Rome, Italy \\ ${ }^{b}$ Center for Atherosclerosis, Policlinico Tor Vergata, Rome, Italy \\ c Unit of Biostatistics, IRCCS Casa Sollievo della Sofferenza, San Giovanni Rotondo, Italy \\ d Department of Experimental Medicine and Surgery, University of Rome Tor Vergata, Rome, Italy \\ e Department of Biopathology and Diagnostic Imaging, University of Rome Tor Vergata, Italy
}

\section{A R T I C L E I N F O}

\section{Article history:}

Received 4 December 2014

Received in revised form 12 January 2015

Accepted 21 January 2015

Available online 29 January 2015

\section{Keywords:}

Atherosclerosis

Cardiovascular disease outcomes

Endothelium-derived factors

Follow up study

Inflammation

\begin{abstract}
A B S T R A C T
Objective: Atherosclerosis disease is a leading cause for mortality and morbidity. The narrowing/rupture of a vulnerable atherosclerotic plaque is accountable for acute cardiovascular events. However, despite of an intensive research, a reliable clinical method which may disclose a vulnerable patient is still unavailable.

Approach and results: We tested the association of ADAM17 (A Disintegrin and Metallo Protease Domain 17) circulating substrates (sICAM-1, sVCAM-1, sIL6R and sTNFR1) with a second major cardiovascular events [MACEs] (cardiovascular death, peripheral artery surgeries, non-fatal myocardial infarction and non-fatal stroke) in 298 patients belonging to the Vascular Diabetes (AVD) study. To evaluate ADAM17 activity we create ADAM17 score through a RECPAM model. Finally we tested the discrimination ability and the reclassification of clinical models.

At follow-up (mean 47 months, range 1-118 months), 55 MACEs occurred (14 nonfatal MI, 14 nonfatal strokes, 17 peripheral artery procedures and 10 cardiovascular deaths) (incidence $=7.8 \%$ person-years). An increased risk for incident events was observed among the high ADAM17 score individuals both in univariable (HR 19.20, 95\% CI 15.82-63.36, p < 0.001) and multivariable analysis (HR 3.42, 95\% CI 1.55 $-7.54, \mathrm{p}<0.001$ ). Finally we found that ADAM17 score significantly increases the prediction accuracy of the Framingham Recurring-Coronary-Heart-Disease-Score, with a significant improvement in discrimination (integrated discrimination improvement $=9 \%, \mathrm{p}=0.012$ ) and correctly reclassifying $10 \%$ of events and $41 \%$ of non-events resulting in a cNRI $=0.51(\mathrm{p}=0.005)$.

Conclusion: We demonstrated a positive role of ADAM17 activity to predicting CV events. We think that an approach that targets strategies beyond classic cardiovascular risk factors control is necessary in individuals with an established vascular atherosclerosis.
\end{abstract}

(c) 2015 Elsevier Ireland Ltd. All rights reserved.
List of abbreviations: ADAM17, A Disintegrin and Metallo Protease Domain 17; MACEs, Major cardiovascular events; CV, Cardiovascular; TIMP3, Tissue inhibitor of metalloproteinase 3; AVD, Athero vascular diabetes; RECPAM, Recursive Partitioning and Amalgamation; RCHD, Recurrent Coronary Heart Disease; IDI, Integrated Discrimination Improvement; RIDI, Relative Integrated Discrimination Improvement; NRI, Net reclassification improvement; PTCA, Transluminal coronary angioplasty; CEA, Carotid endo-arterectomy; OGTT, Oral Glucose Tolerance Test.

* Corresponding author. Center for Atherosclerosis, Department of Systems Medicine, University of Rome Tor Vergata, Via Montpellier 1, 00133 Rome, Italy.

E-mail address: federicm@uniroma2.it (F. Massimo).

\section{Introduction}

Atherosclerosis is the primary cause for coronary artery disease and stroke in the Western World [1].

There is an evolving debate about the value of assessing levels of soluble biomarkers for the prediction of plaques ruptures [2,3]. The challenging point is therefore to identify a soluble biomarker which may be helpful to identify vulnerable patients that despite no clinical evidence of atherosclerosis and no concomitance of several cardiovascular risk factors are very likely to be affected by 
subclinical frail atherosclerotic plaques.

The TNF-alpha Converting Enzyme (TACE), also called A Disintegrin and Metallo Protease Domain 17 (ADAM17), is a type I transmembrane protein that belongs to a superfamily of $\mathrm{Zn}$ dependent metalloproteases. ADAM17 plays a key role in the regulation of the proteolytic release from cellular membranes of some adhesion molecules, cytokines, chemokines, growth factors and their receptors, including ICAM-1, VCAM- 1 , TNF- $\alpha$, TNF receptors I and II, TGF-a, L-selectin, IL-6 receptor and M-CSF receptor 1, affecting downstream signaling and cellular responses [4].

ADAM17 activity is involved in major acute and chronic inflammatory and degenerative diseases such as cancer, inflammatory diseases, Alzheimer and atherosclerosis [4] Interestingly, it has been described that a local ADAM17 activity is correlated to adverse clinical outcomes in acute coronary atherosclerosis setting [5]. Similarly we and others have demonstrated that an unbalanced ADAM 17/tissue inhibitor of metalloproteinase 3 (TIMP3) ratio is characteristic of unstable carotid plaques in diabetic and non diabetic subjects $[6,7]$.

TIMP3 is also an inhibitor of metalloproteinases (MMP) 9 and 14 both involved in plaque destabilization in previous studies [6-8].

Here, we tested whether ADAM17 substrates included in a new single score are associated with cardiovascular events.

Secondarily, we tried to quantify the prediction improvement of a classic algorithm risk score when the assessment of the circulating ADAM17 substrates was added.

\section{Materials and methods}

\subsection{Assessment of ADAM17 substrates}

Serum samples were analyzed by a sandwich enzyme-linked immunosorbent assay (ELISA) method using antimouse antibodies for human soluble tumor necrosis factor receptor 1 (sTNFR1), soluble interleukin 6 receptor (sIL6R), soluble vascular cell adhesion molecule 1 (sVCAM-1), soluble intercellular cell adhesion molecule 1 (sICAM-1) (all from R\&D Systems, Minneapolis, MN) according to manufacturer's instructions.

\subsection{Study participants}

We evaluated baseline ADAM17 activity by dosing its four main circulating substrates levels (sVCAM-1, sICAM-1, sIL6R, sTNFR1) in 298 consecutive participants belonging to the Athero-Vascular Diabetes (AVD) Study which has been described in detail previously [9]. This study was approved by the University of Tor Vergata Institutional Review Board and carried out in accordance with the principles of the Declaration Of Helsinki as revised in 2000.

\subsection{Follow-up}

The patients had a routine follow-up at six months and one year after inclusion, then yearly thereafter. Follow-up outcomes included the development of one major cardiovascular event such as nonfatal stroke, nonfatal myocardial infarction, peripheral vascular surgical procedures and cardiovascular death (fatal stroke or fatal myocardial infarction). Information on nonfatal events was sought from study participants by telephone interviews and confirmed by review of hospital records. In case of fatal events, information was obtained from their primary care physicians or from death certificates.

\subsection{Statistical methods}

Baseline patients' clinical characteristics were reported as mean and standard deviation or frequencies and percentages for continuous and categorical variables, respectively.

Univariable and multivariable time to event analysis were performed using Cox proportional hazards regression model. Risks were reported as hazard ratios (HR) along their 95\% confidence interval $(95 \% \mathrm{CI})$.

The clinical predictive ADAM17 score and the Reclassification methods are described in the online supplementary methods.

A p-value $<0.05$ will be considered as significant. All analyses will be performed using SAS Release 9.3 (SAS Institute, Cary, NC).

\section{Results}

During the follow-up (mean 47 months, range 1-118 months) 55 major adverse cardiovascular events (MACEs) occurred: 14 nonfatal MI, 14 nonfatal strokes, 17 peripheral artery procedures and 10 cardiovascular deaths (incidence of MACEs was 7.8\% personyears). Peripheral artery procedures included 8 PTCA (transluminal coronary angioplasty), 5 CEA (carotid endo-arterectomy) and 4 peripheral stenting placements.

The clinical characteristics of study population according to event or no event are summarized in Table 1. Of note, no patients reported a history of diagnosed diabetes [9]. Therefore, according to the inclusion criteria, all individuals included into the study underwent an Oral Glucose Tolerance Test (OGTT) to detect an unknown diabetes. Consequently, patients with unknown diabetes did not take any specific therapeutic agents. Finally no significant differences in pharmacological therapy emerged between the group of individuals who had a MACES and the group of participants who had not (On-line Table 1).

Crude and age-sex-BMI adjusted second cardiovascular event HRs are reported in Table 2 for the main demographic, clinical and biochemical features.

To create a quantitative risk score which may illustrate the ADAM17 activity on the CV event, we applied a RECPAM model to the circulating levels of ADAM17 substrates (sVCAM-1, sICAM-1, sIL6R, sTNFR1) expressed in tertiles (Fig. 1). The model excluded sIL-6-r because not significantly associated to the event in such a modeling. RECPAM identified 3 homogeneous subgroups of patients in terms of event rate according to specific interactions between s-TNF $\alpha$-rec, sVCAM- 1 and sICAM- 1 levels. Class 1 included the participants with the lower risk to develop the event whereas Class 3 involved patients with the higher risk ( $\mathrm{HR}=6.69, \mathrm{CI} 95 \%$

Table 1

Clinical characteristics of study population.

\begin{tabular}{lll}
\hline & $\begin{array}{l}\text { Patients without MACEs } \\
\left(\mathrm{n}^{\circ} 243\right)\end{array}$ & $\begin{array}{l}\text { Patients with MACEs } \\
\left(\mathrm{n}^{\circ} 55\right)\end{array}$ \\
\hline Age $(\mathrm{y})$ & $58.19 \pm 17.93$ & $66.73 \pm 9.94$ \\
Sex $(\mathrm{m} / \mathrm{f})$ & $148 / 95$ & $44 / 11$ \\
Smokers or ex smokers & $172(71 \%)$ & $51(94 \%)$ \\
$\quad(\mathrm{n}, \%)$ & \\
BMI & $26.53 \pm 4.13$ & $24.43 \pm 4.27$ \\
Diast BP $(\mathrm{mmHg})$ & $80.56 \pm 10.60$ & $80.56 \pm 11.84$ \\
Syst BP $(\mathrm{mmHg})$ & $134.03 \pm 22.61$ & $137.59 \pm 20.92$ \\
Diabetes $(\mathrm{n}, \%)$ & $44(18 \%)$ & $11(20 \%)$ \\
A1c $(\%)$ & $5.51 \pm 0.42$ & $5.69 \pm 0.59$ \\
Tot cholesterol $(\mathrm{mg} / \mathrm{dl})$ & $184.29 \pm 37.69$ & $173.33 \pm 47.34$ \\
LDL cholesterol $(\mathrm{mg} / \mathrm{dl})$ & $116.33 \pm 32.89$ & $109.20 \pm 38.49$ \\
HDL cholesterol $(\mathrm{mg} / \mathrm{dl})$ & $49.96 \pm 14.33$ & $45.98 \pm 13.94$ \\
Triglycerides $(\mathrm{mg} / \mathrm{dl})$ & $124.99 \pm 67.00$ & $138.44 \pm 67.33$ \\
sICAM-1 $(\mathrm{ng} / \mathrm{ml})$ & $285.28 \pm 113.79$ & $357.90 \pm 171.60$ \\
sVCAM-1 $(\mathrm{ng} / \mathrm{ml})$ & $732.94 \pm 386.63$ & $954.97 \pm 393.73$ \\
sIL6R (ng/ml) & $40.33 \pm 12.94$ & $43.06 \pm 10.35$ \\
sTNFR1 (pg/ml) & $1.60 \pm 0.57$ & $1.83 \pm 0.71$ \\
Follow-up (months) & $57.71 \pm 22.19$ & $32.68 \pm 19.98$ \\
\hline
\end{tabular}




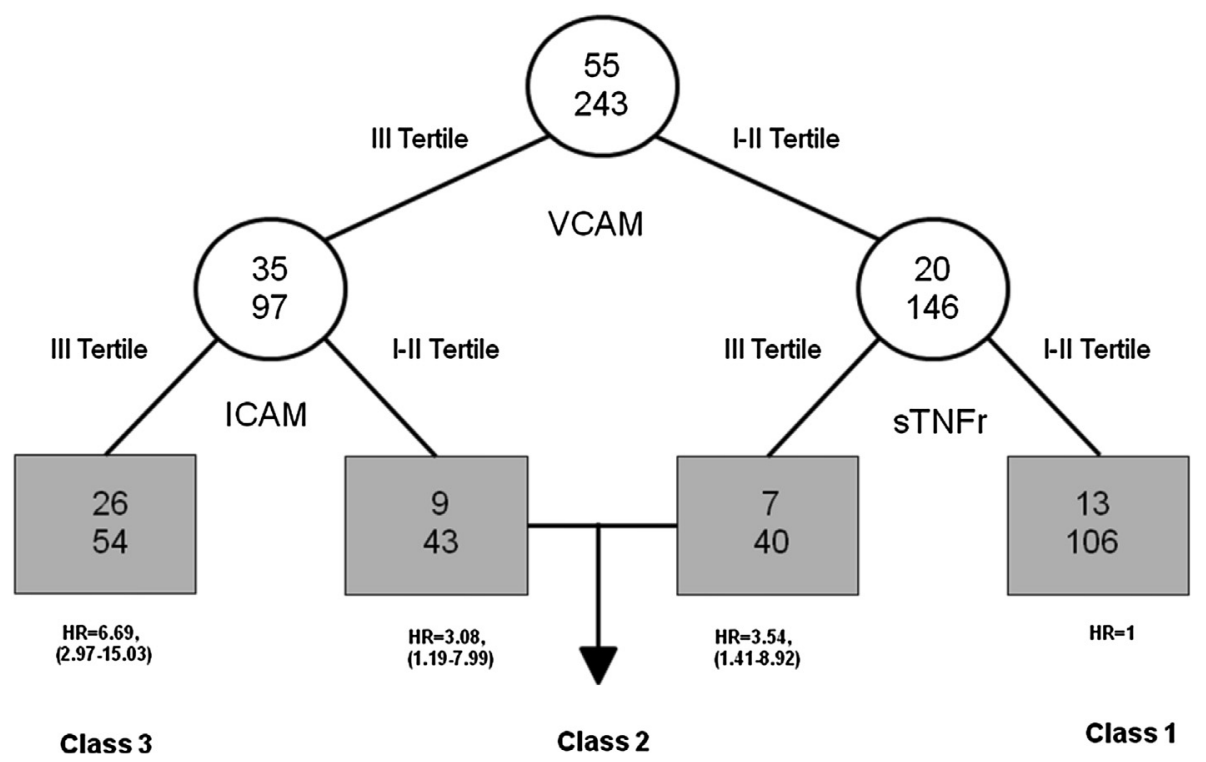

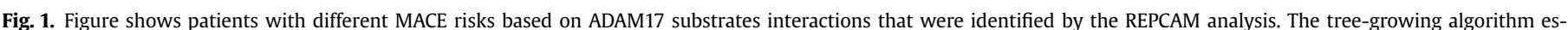

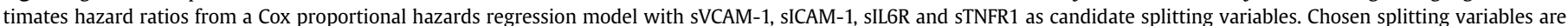

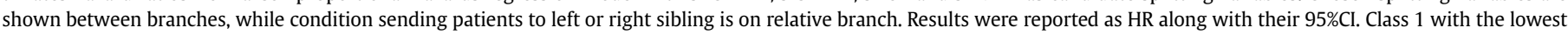

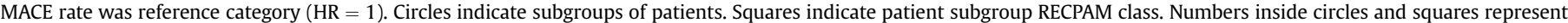
the number of events (top) and the number of non-events (bottom), respectively.

2.97-15.03). Subjects with higher cardiovascular risk (high ADAM17 score level) have the higher tertile of sICAM-1 and the higher tertile of sVCAM-1. On the opposite side, patients with lower cardiovascular risk (low ADAM17 score level) have the lower or medium tertiles of sVCAM-1 and lower or medium tertiles of sTNFR1. All the other individuals have an intermediate risk (medium ADAM17 score level).

In the time to event analysis (Table 2), respect to low ADAM17 score participants, an increased risk for incident events was observed among the high ADAM17 score individuals (KM curves shown in Fig. 2 for display only) both in univariable (HR 19.20, 95\%
CI 15.82-63.36, $\mathrm{p}<0.001$ ) and multivariable analysis (HR 3.42, 95\% CI 1.55-7.54, $\mathrm{p}<0.001$ ) after adjusting for age, sex and BMI.

Next, we tested if the addition of ADAM17 score to the Framingham Recurring Coronary Heart Disease (RCHD) model [10]. It is a well established predictive risk model; thus we assessed if the addition of ADAM17 score could improve the 4-year discriminative prediction of MACEs. We found that ADAM17 score significantly increases the prediction accuracy. The survival C-statistic of RCHD score was $0.74(95 \% \mathrm{CI}=0.67-0.81)$ and such a model resulted calibrated (HL, $\mathrm{p}=0.91$ ). Addition of the ADAM17 score into this model (calibration HL, $\mathrm{p}=0.95$ ) led to significant increase of

Table 2

Univariable and multivariable associations between clinical parameters and MACEs.

\begin{tabular}{|c|c|c|c|c|c|c|}
\hline \multirow[t]{2}{*}{ Variable } & \multicolumn{3}{|c|}{ Univariable analysis } & \multicolumn{3}{|c|}{ Multivariable analysis $^{a}$} \\
\hline & HR & $95 \% \mathrm{CI}$ & $\mathrm{p}$ & HR & $95 \% \mathrm{CI}$ & $\mathrm{p}$ \\
\hline \multicolumn{7}{|l|}{ Sex } \\
\hline Male & 3.023 & $1.561-5.853$ & 0.001 & 4.326 & $0.947-19.766$ & 0.059 \\
\hline Female & 1 & & & 1 & & \\
\hline Age & 1.031 & $1.013-1.050$ & $<0.001$ & 1.011 & $0.954-1.071$ & 0.713 \\
\hline BMI & 1.052 & $0.991-1.116$ & 0.097 & 1.123 & $0.848-1.487$ & 0.417 \\
\hline \multicolumn{7}{|l|}{ Smoke } \\
\hline Ex or current & 3.665 & $1.846-7.275$ & $<0.001$ & 1.05 & $0.285-3.867$ & 0.942 \\
\hline Never & 1 & & & 1 & & \\
\hline Systolic BP & 1.006 & $0.994-1.017$ & 0.332 & 1.007 & $0.971-1.043$ & 0.718 \\
\hline Diastolic BP & 1.002 & $0.978-1.027$ & 0.855 & 0.99 & $0.919-1.065$ & 0.779 \\
\hline TOT Cholesterol & 0.993 & $0.987-1.000$ & 0.054 & 0.997 & $0.943-1.054$ & 0.924 \\
\hline HDL Chol & 0.977 & $0.958-0.997$ & 0.026 & 1.007 & $0.948-1.069$ & 0.829 \\
\hline LDL Chol & 0.994 & $0.986-1.002$ & 0.12 & 0.982 & $0.929-1.038$ & 0.511 \\
\hline Triglycerides & 1.003 & $1.000-1.006$ & 0.083 & 0.969 & $0.942-0.996$ & 0.025 \\
\hline A1c & 2.202 & $1.346-3.601$ & 0.002 & 4.505 & $0.810-25.068$ & 0.086 \\
\hline sIL6R & 1.016 & $0.997-1.036$ & 0.099 & 0.76 & $0.580-0.996$ & 0.047 \\
\hline sTNFR1 & 1.7 & $1.152-2.507$ & 0.007 & 22.255 & $1.79-276.76$ & 0.016 \\
\hline sVCAM1 & 1.001 & $1.001-1.001$ & $<0.001$ & 1.001 & $0.997-1.005$ & 0.692 \\
\hline sICAM-1 & 1.003 & $1.002-1.004$ & $<0.001$ & 1.003 & $0.994-1.013$ & 0.512 \\
\hline \multicolumn{7}{|l|}{ ADAM17 score } \\
\hline High ADAM17 score & 19.2 & $5.819-63.361$ & $<0.001$ & 3.415 & $1.548-7.536$ & 0.002 \\
\hline Medium ADAM17 score & 12.52 & $3.737-41.933$ & $<0.001$ & 1.918 & $0.857-4.291$ & 0.113 \\
\hline Low ADAM17 score & 1 & & & 1 & & \\
\hline
\end{tabular}

\footnotetext{
${ }^{\text {a }}$ Adjusting for patient's age, sex and BMI.
} 


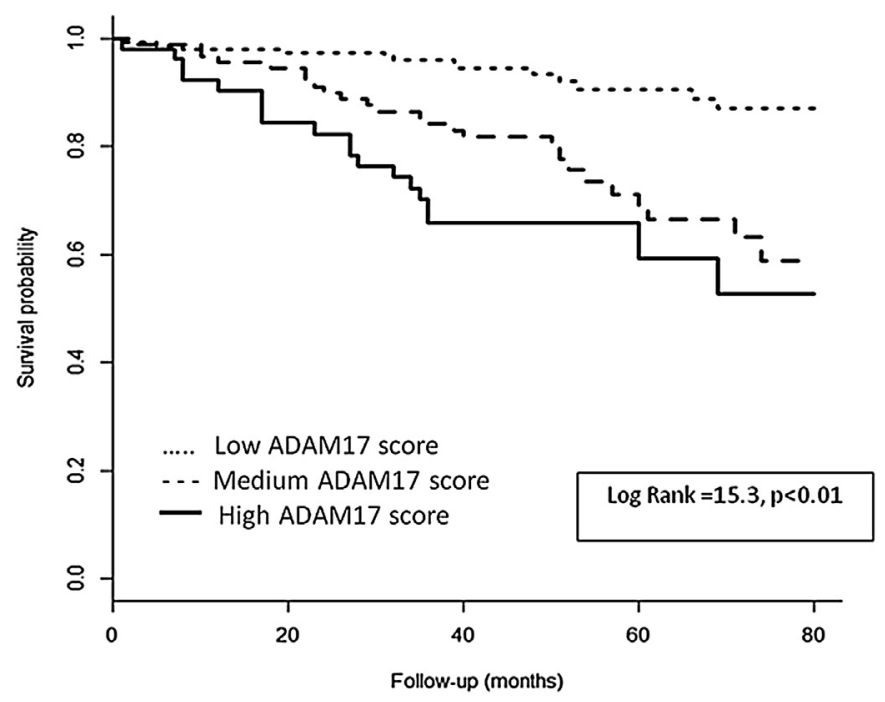

Fig. 2. Kaplan-Meier curves for ADAM17 score classes. Log Rank: 15.3, p < 0.01 .

survival C-statistic ( $\mathrm{p}=0.018)$ to $0.80(95 \% \mathrm{CI}=0.70-0.85)$ as well as statistically significant improvement in discrimination evaluated by IDI $=9 \%(95 \% \mathrm{CI}=4 \%-14 \%, \mathrm{p}=0.012)$ and $\mathrm{RIDI}=39 \%(95 \% \mathrm{CI}$ : $7 \%-74 \%, \mathrm{p}=0.013)$. Moreover, the introduction of ADAM17 score into the model correctly reclassified $10 \%$ of events and $41 \%$ of nonevents resulting in a cNRI $=0.51(95 \% \mathrm{CI}=0.10-0.79, \mathrm{p}=0.005)$.

\section{Discussion}

During the past decade the identification of atherosclerotic vulnerable plaques, likely to cause an acute event in the future, has been a topic of intensive research. Several noninvasive modalities has been evaluated, but, unfortunately, a method that can properly assess all the aspects of plaque and based on non invasive approach either at imaging or biochemical level, is still unavailable [11]. We found that increased circulating levels of ADAM17 substrates were associated to a significant higher rate of second cardiovascular events in a cohort of patients with established vascular atherosclerosis. Although we have not explored ADAM17 protease activity directly in vivo it is conceivable that the increase of its substrates in the circulation depends from increased enzymatic activity at local inflammatory sites. Therefore, to study ADAM17 activity in an atherosclerotic setting we decided to evaluate, among its many substrates [12,13], those that were already independently implicated in severity or complications of atherosclerosis [14], but never with a combined approach. We reasoned that local over-activity of ADAM17 may weaken atherosclerotic plaque, causing their rupture, as a consequence of enzyme activation promoted by well known cardiovascular risk factors such as hyperglycemia, hyperinsulinemia and oxidized LDL and consequent increase in intraplaque inflammatory cells trafficking [7,14-16].

We have chosen to analyze ADAM17 substrates since they also unify the effect of different risk factors (such as insulin resistance/ diabetes) on endothelial function and the consequent effect on atherosclerosis plaque evolution [17-20].

To explore ADAM17 in vivo activity we created a simple 3 levels score by RECPAM model that was able to identify distinct and homogeneous subgroups of individuals in terms of event rate and, therefore, those with very high cardiovascular risk. Our ADAM17 score is composed by circulating levels of sTNFR1, sVCAM-1, sICAM1 but not sIL6R because the latter was not significantly associated to the events. This is surprising since sIL6R has been related to cardiovascular mortality [21]. However, it has been recently described that the most common functional variant in IL6R (Asp358Ala) is unrelated to the common CVD risk factors and it is associated in a dose-dependent manner with a decreased concentration of C-reactive protein and fibrinogen, and might consequently dampen the systemic inflammation [22]. This result supports the hypothesis that IL6R may play different roles depending on the different genetic variant.

The Framingham risk score remains the most widely used tool for assessment of risk prediction of CV events [23]. Conventional risk factors in the Framingham risk score such as age, male sex, hypercholesterolemia, hypertension, and smoking, account for most of the risk of CVD and have been the basis of risk assessment for decades. However, approximately one-third of individuals with 0 or 1 risk factor develop coronary heart diseases [24,25] and up to $40 \%$ of individuals with cholesterol levels below the population average die from CHD [26]. Furthermore, many CV events occur in patients treated with statin therapy.

As such, a wide list of biomarkers, genetic polymorphism arrays, and direct imaging of subclinical atherosclerosis with coronary artery calcium or carotid intima-media thickness have been investigated for refinement of risk assessment and preventive therapy allocation.

Individuals in secondary prevention have already a diffuse vascular atherosclerosis; therefore the use of a risk algorithm which does not include the plaques biology evaluation, or at least a surrogate, is a particular limitation. In fact, using only the classic cardiovascular risk factors we treat at the same manner patients with an initial vascular inflammation and individuals with an overt cardiovascular atherosclerosis. Differently, our data, including endothelial activation/inflammatory markers, may increase our ability to identify the vulnerable patient. Obviously other factors pointing to assess the thrombotic potential or the digestion of the necrotic core in the atherosclerotic plaque might be added in future studies.

We found a significant association between ADAM17 score and $\mathrm{CV}$ events both in univariable and multivariable analysis. To confirm the strength of our finding we incorporated the ADAM17 score into Framingham RCHD score [10] to improve its ability in predicting a second CV event. ADAM17 score substantively improved the Framingham RCHD. We showed a significant discrimination and reclassification improvement for CV events when ADAM17 score was assessed in addition to classical risk factors. The combined incorporation of the 3 biomarkers as a single covariate improved the c-statistic and reclassification metrics. Our result is statistically significant but rather faint. This point may be an important limitation but certainly is not surprising. In fact it has been already demonstrated that in particular setting of patients the incorporation of external risk factors into the Framingham score improves global CVD risk prediction, and when it happens, this improvement is frequently modest $[27,28]$ even when the added biomarker (hsCRP) is strongly associated to CV risk [29]. Noteworthy the observed improvement is not only statistically significant but also clinically relevant allowing correct reclassification of large proportion of patients both with and without events. Nevertheless, our present finding needs replication in large prospective studies before it can be considered as established.

Our results have some limitations. First, the lack of a second cohort for an independent validation, although a replication of our finding is not easy because of the very particular vascular condition of the study population. Every patient had a non fatal MACE and no apparent record of DM2. However, to strengthen our results we adopted a permutation analysis for RECPAM model and used a bootstrap approach for the reclassification metrics, thus avoiding to produce over-optimistic results and obtaining a robust internal 
validation.

Second, we acknowledge that a composite end-point comprising different cardiovascular events may be related to different pathogenic backgrounds. We cannot exclude, in fact, that some severe events may be unrelated to "vulnerable plaques" such as embolic stroke or a peripheral vascular surgical procedures for a slow narrowing of an atherosclerotic plaque. Though we recognized that further studies using unique clinical events as independent endpoints are likely to allow a deeper understanding of the biology of atherosclerotic plaque vulnerability, our results suggest that the use of ADAM17 substrates altogether may be a short come to trace the increased activity of the inflammatory and remodeling processes involved in plaque destabilization [30,31]. Whether the predictive role of ADAM17 score may be extended to clinical situations involving patients with a more moderate cardiovascular risk remains to be determined.

\section{Significance}

Our results suggest that measuring ADAM17 activity may help to predict major cardiovascular events in subjects with an established vascular atherosclerosis. To evaluate ADAM17 activity we dosed its four main substrates levels (sVCAM-1, sICAM-1, sIL6R, sTNFR1) in 298 consecutive participants with an established clinical vascular atherosclerosis, who were followed for MACEs (mean 47 months, range 1-118 months). To create a quantitative risk score which may illustrate the ADAM17 activity on the CV event we applied a RECPAM model. RECPAM identified 3 homogeneous subgroups of patients in terms of event risk.

An increased risk for incident events was observed among the high ADAM17 score individuals both in univariable and multivariable analysis. Finally we showed a significant enhancement of the Framingham risk score by adding ADAM17 activity to the classical risk factors. We conclude that in individuals with an established vascular atherosclerosis an approach that targets strategies beyond classic cardiovascular risk factors control is helpful.

\section{Source of funding}

This manuscript was funded by PRIN 20123BJ89E and Progetto SID 2013 to M.F.

\section{Disclosures}

Authors declare that they have neither financial nor competing interests.

\section{Conflict of interest}

Authors declare that they have no conflicts of interest.

\section{Acknowledgement}

None.

\section{Appendix A. Supplementary data}

Supplementary data related to this article can be found at http:// dx.doi.org/10.1016/j.atherosclerosis.2015.01.029.

\section{References}

[1] C.K. Glass, J.L. Witztum, Atherosclerosis, the road ahead, Cell 104 (2001) $503-516$

[2] G.L. Myers, R.H. Christenson, M. Cushman, C.M. Ballantyne, G.R. Cooper,
C.M. Pfeiffer, S.M. Grundy, D.R. Labarthe, D. Levy, N. Rifai, P.W. Wilson, National Academy of Clinical Biochemistry Laboratory Medicine Practice Guidelines: emerging biomarkers for primary prevention of cardiovascular diseases, Clin. Chem. 55 (2) (2009) 378-384.

[3] R. Virmani, F.D. Kolodgie, A.P. Burke, A. Farb, S.M. Schwartz, Lessons from sudden coronary death: a comprehensive morphological classification scheme for atherosclerotic lesions, Arterioscler. Thromb. Vasc. Biol. 20 (2000) $1262-1275$.

[4] R. Menghini, L. Fiorentino, V. Casagrande, R. Lauro, M. Federici, The role of ADAM17 in metabolic inflammation, Atherosclerosis 228 (1) (2013) 12-17.

[5] M. Satoh, Y. Ishikawa, T. Itoh, Y. Minami, Y. Takahashi, M. Nakamura, The expression of TNF-alpha converting enzyme at the site of ruptured plaques in patients with acute myocardial infarction, Eur. J. Clin. Investig. 38 (2) (2008) 97-105.

[6] A. Müller, S.D. Krämer, R. Meletta, et al., Gene expression levels of matrix metalloproteinases in human atherosclerotic plaques and evaluation of radiolabeled inhibitors as imaging agents for plaque vulnerability, Nucl. Med. Biol. 41 (7) (2011) 562-569.

[7] M. Cardellini, R. Menghini, A. Luzi, F. Davato, I. Cardolini, R. D'Alfonso, P. Gentileschi, S. Rizza, M.A. Marini, O. Porzio, D. Lauro, P. Sbraccia, R. Lauro, M. Federici, Decreased IRS2 and TIMP3 expression in monocytes from offspring of type 2 diabetic patients is correlated with insulin resistance and increased intima-media thickness, Diabetes 60 (12) (2011) 3265-3270.

[8] R. Khokha, A. Murthy, A. Weiss, Metalloproteinases and their natural inhibitors in inflammation and immunity, Nat. Rev. Immunol. 13 (9) (2013) 649-665.

[9] S. Rizza, M. Copetti, M. Cardellini, O. Porzio, A. Luzi, C. Pecchioli, E. Martelli, A. Valentini, A. Ippoliti, F. Romeo, F. Pellegrini, D. Lauro, R. Lauro, M. Federici, Atherosclerosis severity but not undiagnosed diabetes predicts new cardiovascular events of subjects in secondary cardiovascular prevention, Atherosclerosis 223 (2) (2012) 448-453.

[10] R.B. D'Agostino, M.W. Russell, D.M. Huse, R.C. Ellison, H. Silbershatz, P.W. Wilson, S.C. Hartz, Primary and subsequent coronary risk appraisal: new results from the Framingham study, Am. Heart J. 139 (2 Pt 1) (2000) 272-281.

[11] N. Tahara, J. Mukherjee, H.J. de Haas, et al., 2-deoxy-2-[(18)F]fluoro-dmannose positron emission tomography imaging in atherosclerosis, Nat. Med. 20 (2) (2014) 215-219.

[12] C.M. Overall, C.P. Blobel, In search of partners: linking extracellular proteases to substrates, Nat. Rev. Mol. Cell Biol. 8 (2007) 245-257.

[13] Akira Takaguri, Keita Kimura, Akinari Hinoki, Allison M. Bourne, Michael V. Autieri, Satoru Eguchi, A disintegrin and metalloprotease 17 mediates neointimal hyperplasia in vasculature, Hypertension 57 (4) (2011) 841-845.

[14] P. Gutwein, M.S. Abdel-Bakky, A. Schramme, K. Doberstein, N. Kämpfer-Kolb, K. Amann, I.A. Hauser, N. Obermüller, C. Bartel, A.A. Abdel-Aziz, S.M. El Sayed el, J. Pfeilschifter, CXCL16 is expressed in podocytes and acts as a scavenger receptor for oxidized low-density lipoprotein, Am. J. Pathol. 174 (6) (2009) 2061-2072.

[15] X.Q Zhao, M.W. Zhang, F. Wang Y.X. Zhao, JJ. Li, X.P. Wang P.L. Bu, J.M. Yang, X.L. Liu, M.X. Zhang, F. Gao, C. Zhang, Y. Zhang, CRP enhances soluble LOX-1 release from macrophages by activating TNF- $\alpha$ converting enzyme, J. Lipid Res. 52 (5) (2011) 923-933.

[16] K. Reiss, I. Cornelsen, M. Husmann, G. Gimpl, S. Bhakdi, Unsaturated fatty acids drive disintegrin and metalloproteinase (ADAM)-dependent cell adhesion, proliferation, and migration by modulating membrane fluidity, J. Biol. Chem. 286 (30) (2011) 26931-26942.

[17] V. Casagrande, R. Menghini, S. Menini, et al., Overexpression of tissue inhibitor of metalloproteinase 3 in macrophages reduces atherosclerosis in low-density lipoprotein receptor knockout mice, Arterioscler. Thromb. Vasc. Biol. 32 (1) (2012) 74-81.

[18] L. Fiorentino, A. Vivanti, M. Cavalera, V. Marzano, M. Ronci, M. Fabrizi, S. Menini, G. Pugliese, R. Menghini, R. Khokha, R. Lauro, A. Urbani, M. Federici, Increased tumor necrosis factor alpha-converting enzyme activity induces insulin resistance and hepatosteatosis in mice, Hepatology 51 (1) (2010) $103-110$.

[19] Stöhr R, Cavalera M, Menini S, Mavilio M, Casagrande V, Rossi C, Urbani A Cardellini M, Pugliese G, Menghini R, Federici M, Loss of TIMP3 exacerbates atherosclerosis in ApoE null mice, Atherosclerosis 201, 235(2), 438-443.

[20] A.C. Newby, Proteinases and plaque rupture: unblocking the road to translation, Curr. Opin. Lipidol. 25 (5) (2014) 358-366.

[21] The IBC 50K CAD Consortium, Large-scale gene-centric analysis identifies novel variants for coronary artery disease, PLoS Genet. 7 (2011) e1002260.

[22] The Interleukin-6 Receptor Mendelian Randomisation Analysis (IL6R MR) Consortium, The interleukin-6 receptor as a target for prevention of coronary heart disease: a mendelian randomisation analysis, The Lancet 379 (2012) 1214-1224.

[23] S.M. Grundy, G. Balady, M. Criqui, G. Fletcher, P. Greenland, L Hiratzka, N. Houston-Miller, P. Kris-Etherton, H. Krumholz, J. LaRosa, I.S. Ockene, T.A. Pearson, J. Reed, R. Washington, S.C. Smith Jr., Primary prevention of coronary heart disease: guidance from Framingham: a statement for healthcare professionals from the AHA Task Force on Risk Reduction. American Heart Association, Circulation 97 (1998) 1876-1887.

[24] P. Greenland, M.D. Knoll, J. Stamler, J.D. Neaton, A.R. Dyer, D.B. Garside, P.W. Wilson, Major risk factors as antecedents of fatal and nonfatal coronary heart disease events, JAMA 290 (2003) 891-897.

[25] U.N. Khot, M.B. Khot, C.T. Bajzer, S.K. Sapp, E.M. Ohman, S.J. Brener, S.G. Ellis, 
A.M. Lincoff, E.J. Topol, Prevalence of conventional risk factors in patients with coronary heart disease, JAMA 290 (2003) 898-904.

[26] S.C. Smith Jr., Current and future directions of cardiovascular risk prediction, Am. J. Cardiol. 97 (2006) 28A-32A.

[27] P.M. Ridker, J.E. Buring, N. Rifai, N.R. Cook, Development and validation of improved algorithms for the assessment of global cardiovascular risk in women: the Reynolds risk score, JAMA 297 (2007) 611-619.

[28] B. Zethelius, L. Berglund, J. Sundstrom, E. Ingelsson, S. Basu, A. Larsson, P. Venge, J. Arnlöv, Use of multiple biomarkers to improve the prediction of death from cardiovascularcauses, N. Engl. J. Med. 358 (2008) 2107-2116.
[29] Yousuf O, Mohanty B, Martin S, Joshi PH, Blaha MJ, Nasir K, Blumenthal RS, Budoff MJ, High-sensitivity C-reactive protein and cardiovascular disease: resolute belief or an elusive link? JACC, 201, 5, 397-408.

[30] M. Naghavi, P. Libby, E. Falk, et al., From vulnerable plaque to vulnerable patient: a call for new definitions and risk assessment strategies: part II Circulation 108 (15) (2003) 1772-1778, 14

[31] M. Naghavi, P. Libby, E. Falk, et al., From vulnerable plaque to vulnerable patient: a call for new definitions and risk assessment strategies: part I, Circulation 108 (14) (2003) 1664-1672, 7. 